\title{
Primeiro registro do gênero Myriostoma (Geastraceae, Basidionycota) no Parque Estadual das Fontes do Ipiranga (PEFI), São Paulo, SP, Brasil
}

\author{
Larissa Trierveiler-Pereira ${ }^{1,3}$ e Adriana de Mello Gugliotta
}

Recebido: 8 maio 2019; aceito: 10 julho 2019

Como citar: Trierveiler-Pereira, L. \& Gugliotta, A.M. 2020. Primeiro registro do gênero Myriostoma (Geastraceae, Basidiomycota) no Parque Estadual das Fontes do Ipiranga (PEFI), São Paulo, SP, Brasil. Hoehnea 47: e512019. http:// dx.doi.org/10.1590/2236-8906-51/2019.

ABSTRACT - (First record of Myriostoma (Geastraceae, Basidiomycota) in the Parque Estadual das Fontes do Ipiranga (PEFI), São Paulo, São Paulo State, Brazil). Myriostoma is an easily identifiable genus due to its basidiome with multiple stalks and ostioles in the endoperidium. Its occurrence in South America is limited to Brazil and Argentina, but the basidiomes are not abundant and easily found in nature. The aim of this study is to report for the first time the oceurrence of Myriostoma calongei in the Parque Estadual das Fontes do lpiranga, São Paulo, since the genus was reported only once from the State. Keywords: earthstar fungi, neotropical fungi, taxonomy

RESUMO - (Primeiro registro do gênero Myriostoma (Geastraceae, Basidiomycota) no Parque Estadual das Fontes do Ipiranga (PEFI), São Paulo, SP, Brasil). O gênero Myriostoma e facilmente identificado devido aos basidiomas com múltiplas colunas e ostíolos do endoperídio. A ocorrência do gênero na America do Sul está lìmitada ao Brasíl e Argentina, porém os basidiomas não são abundantes e facilmente encontrados na natureza. O objetivo do presente trabalho é registrar pela primeira vez a ocorrência de Myriostomá calongei no Parque Estadual das Fontes do Ipiranga, cidade de São Paulo, sendo que o gênero havia sido registrado apenas uma vez para o Estado de São Paulo.

Palavras-chave: fungos estrela-da-terra, fungos neotropicais, taxonomia

\section{Introdução}

Os membros da família Geastraceae Corda, também conhecidos como estrelas-da-terra, formam basidiomas do tipo gasteroide, ou seja, permanecem fechados até a maturação completa dos esporos e não possuem mecanismos ativos de liberação de esporos. Quando imaturos, os basidiomas são enclausurados, globosos a subglobosos. Após a maturidade dos esporos, as capas externas do basidiomas (exoperídio) se abrem de forma estrelada revelando o saco esporífero, denominado endoperídio. $O$ endoperídio pode ser séssil (estar ligado diretamente ao exoperídio), ou estipitado, sendo elevado do exoperídio por uma ou mais estruturas colunares. A gleba, porção fértil onde são produzidos os esporos sexuais, é pulverulenta e de coloração escura. Os esporos são liberados por um ou diversos orifícios (ostíolos) que se encontram na superfície do endoperídio (Sunhede 1989).

As espécies de Myriostoma, popularmente conhecidas como fungos-pote-de-pimenta, são caracterizadas por produzirem basidiomas de grande tamanho (até aprox. $10 \mathrm{~cm}$ de altura) quando comparados com basidiomas de Geastrum Pers. que geralmente medem de 1 a $5 \mathrm{~cm}$ de altura. $\mathrm{O}$ exoperídio é arqueado e robusto, de coloração marrom, e endoperídio marrom acinzentado podendo exibir um aspecto metálico. O endoperídio é multiestipitado, sendo sustentado por um agrupamento de pequenas colunas amareladas; e a superfície do endoperídio

1. Faculdadé de Tecnologia de Itapetininga, Rua João Vieira Camargo, 104, Vila Barth, 18205-600 Itapetininga, SP, Brasil

2. Instituto de Botânica, Núcleo de Pesquisa em Micologia, Av. Miguel Stefano, 3.687, Águá Funda, 04301-902 São Paulo, SP, Brasil

3. Autor para correspondência: Lt_pereira@yahoo.com.br 
é multiostiolar, ou seja, possui diversos orifícios. A gleba é formada por hifas com pequenos espinhos e protuberâncias na superfície, enquanto os esporos são globosos, ornamentados, reticulados, amarelados à amarronzados (Calonge 1998, Miller \& Miller 1988, Bates 2004).

Myriostoma foi considerado monoespecífico por diversos autores (e.g. Miller \& Miller 1988, Sunhede 1989, Kirk et al. 2008), sendo a espécie M. coliforme (Dicks.) Corda a única aceita com distribuição cosmopolita. Entretanto, a análise de dados moleculares aliada à estudos morfológicos indica que mais espécies devem ser incluídas no gênero (Sousa et al. 2017).

No mês de abril de 2019, basidiomas de Myriostoma foram encontrados no Parque Estadual das Fontes do Ipiranga (São Paulo) e identificados como M. calongei. O objetivo desse trabalho é de registrar a primeira ocorrência dessa espécie fúngica na área de coleta.

\section{Material e métodos}

O Parque Estadual das Fontes do Ipiranga (PEFI) está localizado no Bairro Água Funda, região sudeste do município de São Paulo, SP (coordenadas geográficas $23^{\circ} 361^{\prime} \mathrm{S}$ e $\left.46^{\circ} 38^{\prime} \mathrm{W}\right)$. Possui uma área aproximada de 549,31 hectares com altitude média de 798 metros (Bicudo et al. 2002).

Os basidiomas foram fotografados in situ e coletados do solo manualmente para posterior análise laboratorial. Os basidiomas foram secos em estufa elétrica de circulação de ar e examinados seguindo técnicas tradicionais do estudo de gasteromicetos (Calonge 1998). Materiais brasileiros de $M$. calongei foram recentemente descritos por Sousa et al. (2017), dessa maneira, indicamos essa referência para a descrição da espécie. O material estudado encontra-se depositado no Herbário SP (Thiers, 2019).

\section{Resultados e Discussão}

Myriostoma calongei Baseia, J.O. Sousa \& M.P. Martín, PLoS ONE 12(6): e0177873, 9 (2017).

Figuras 1-4

Descrição da espécie: Sousa et al. (2017).

Material examinado: BRASIL. SÃo PAULO: São Paulo, Parque Estadual das Fontes do Ipiranga, 12-IV-2019,
A.M. Gugliotta 1630 (SP 499092); ibid., 30-IV-2019, A.M. Gugliotta 1631 (SP 499093).

Material adicional examinado: BRASIL. Rio GRANDE Do Sul: Viamão, área rural, 17-IV-2011, L. Trierveiler-Pereira LTP202 (ICN 177080).

Habitat: basidiomas crescem gregários sobre solo.

Distribuição no Brasil: Rio Grande do Sul (Homrich 1973, Trierveiler-Pereira et al. 2018), São Paulo (Homrich 1973), Paraíba e Pernambuco (Baseia \& Galvão 2002) - todos os registros como M. coliforme; Rio Grande do Norte (Sousa et al. 2017).

Comentários: Myriostoma calongei foi descrita recentemente, utilizando a abordagem de taxonomia integrativa, unindo dados de caracteres morfológicos com dados moleculares. A espécie é caracterizada pela superfície do endoperídio áspera e verrucosa (figura 3) devido a presença de fascículos hifais triangulares. Os basidiósporos são amarelados, subglobosos, 5,9-8,7 × 5,6-7,6 $\mu \mathrm{m}$, com ornamentação reticulada, sendo que as ornamentações são formadas por colunas confluentes de 1,0-2,3 $\mu \mathrm{m}$ de altura.

De acordo com os autores (Sousa et al. 2017), a espécie é encontrada no Brasil e Argentina, sendo que a espécie $M$. coliforme é europeia. Outra espécie neotropical, M. areolatum M.P. Martín, J.O. Sousa \& Baseia, ocorre na Costa Rica e pode ser diferenciada de $M$. calongei pelos ostíolos do endoperídio que são aureolados e tubulares (Calonge \& Mata 2006, Sousa et al. 2017).

Um espécime de Myriostoma, identificado como M. coliforme, foi coletado por W. Hoehne na cidade de Limeira (São Paulo) em 1956. Entretanto, o material se encontra depositado no Herbário NY (SpeciesLink, 2019), não havendo assim materiais de Myriostoma coletados no Estado de São Paulo depositados em herbários brasileiros.

No Parque Estadual das Fontes do Ipiranga, um primeiro levantamento da diversidade de fungos macroscópicos foi apresentado por Bononi et al. (1981), porém as únicas espécies de Geastraceae reportadas foram: Geastrum ambiguum Mont., G. rufescens Pers., G. schweinitzii (Berk. \& M.A. Curtis) Zeller e G. subiculosum Cooke \& Massee. No presente trabalho registramos pela primeira vez a ocorrência do gênero Myriostoma no PEFI, e o segundo registro para o Estado de São Paulo, após mais de sessenta anos. 

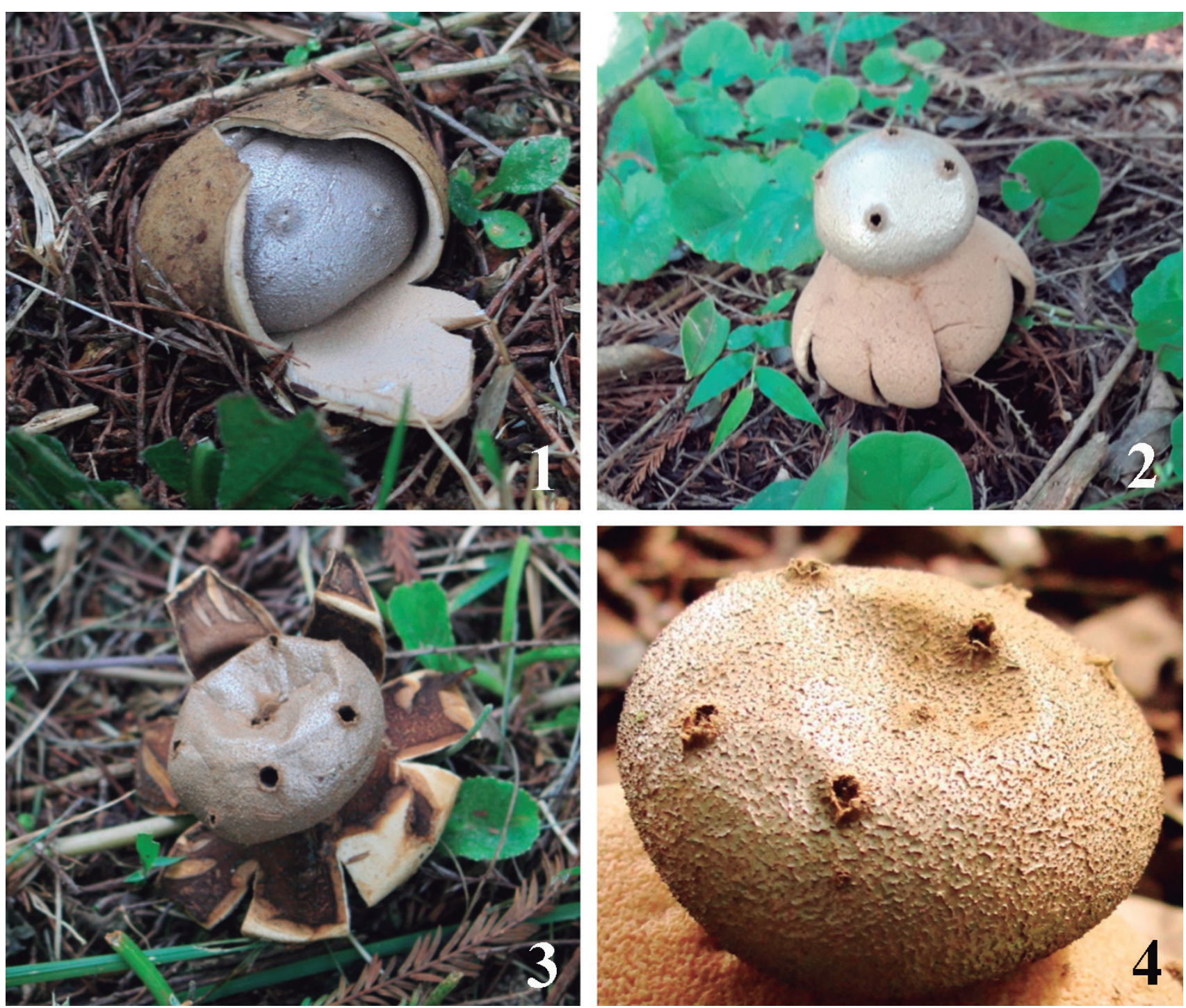

Figuras 1-4. Myriostoma calongei. 1-3. Basidiomas coletados no PEFI em diferentes estágios de maturação (1 e 3: SP 499093; 2: SP 499092). 4. Detalhe do endoperídio verrucoso (ICN 177080). Fotografias são autoria de: 1. Shirlei Soares Dassi; 2 . Inês Cordeiro; 3. Adriana M. Gugliotta; 4. Larissa Trierveiler-Pereira.

Figures 1-4. Myriostoma calongei. 1-3. Basidiomes collected in the PEFI at different development stages (1 and 3: SP 499093; 2: SP 499092). 3. Detail of the verrucose endoperidium (ICN 177080). Photographs by: 1. Shirlei Soares Dassi; 2. Inês Cordeiro; 3 . Adriana M. Gugliotta; 4. Larissa Trierveiler-Pereira.

\section{Agradecimentos}

As autoras agradecem ao Instituto de Botânica pelo apoio institucional e à Fundação de Amparo à Pesquisa do Estado de São Paulo (FAPESP) pelo apoio financeiro concedido (processo 2017/50341-0). Ainda, agradecem à Shirlei Soares Dassi e Inês Cordeiro pelas fotografias realizadas in situ (figuras 1 e 2, respectivamente).

\section{Literatura citada}

Baseia, I.G. \& Galvão, T.C.O. 2002. Some interesting Gasteromycetes (Basidiomycota) in dry areas from Northeastern Brazil. Acta Botanica Brasilica 16: 1-8.
Bates, S.T. 2004. Arizona members of the Geastraceae and Lycoperdaceae (Basidiomycota, Fungi). Tese de Doutorado, Arizona State University, Tempe.

Bicudo, D.C., Forti, M.C. \& Bicudo, C.E.M. 2002. Parque Estadual das Fontes do Ipiranga (PEFI): unidade de conservação que resiste à urbanização de São Paulo. Secretaria do Meio Ambiente do Estado de São Paulo, São Paulo.

Bononi, V.L., Trufem, S.F.B. \& Grandi, R.A.P. 1981. Fungos macroscópicos do Parque Estadual das Fontes do Ipiranga, São Paulo, Brasil, depositados no Herbário do Instituto de Botânica. Rickia 9: 37-53. 
Calonge, F.D. 1998. Flora Mycologica Iberica. v. 3. Gasteromycetes, I. Lycoperdales, Nidulariales, Phallales, Sclerodermatales, Tulostomatales. J. Carmer, Madri, Berlim e Estugarda.

Calonge, F.D. \& Mata, M. 2006. Adiciones y correcciones al catálogo de Gasteromycetes de Costa Rica. Boletín de la Sociedad Micológica de Madrid 30: 111-119.

Homrich, M.H. 1973. Notas sobre Myriostoma coliforme Desvaux (Lycoperdaceae). Iheringia, Série Botânica 18: 80-89.

Kirk, P.M., Cannon, P.F., Minter, D.W. \& Stalpers, J.A. 2008. Dictionary of Fungi. 10 ed. CBS, Utrecht.

Miller, O.K. \& Miller, H.H. 1988. Gasteromycetes. Morphological and development features with keys to orders, families, and genera. Mad River, Eureka.
Sousa, J.O., Suz, L.M., García, M.A., Alfredo, D.S., Conrado, L.M., Marinho, P., Ainsworth, A.M., Baseia, I.G. \& Martín, M.P. 2017. More than one fungus in the pepper pot: integrative taxonomy unmasks hidden species within Myriostoma coliforme (Geastraceae, Basidiomycota). PLoS ONE 12: e0177873

SpeciesLink. 2019. Disponível em http://splink.cria.org. br/ (acesso em 30-IV- 2019).

Sunhede, S. 1989. Geastraceae (Basidiomycotina): Morphology, ecology and systematics with special emphasis on the North European species. Synopsis Fungorum. v. 1. Fungiflora, Oslo.

Thiers, B. 2019. Index Herbariorum. Disponível em http:// sweetgum.nybg.org/science/ih/ (acesso em 30-IV-2019).

Trierveiler-Pereira, L., Honaiser, L.P. \& Silveira, R.M.B. 2018. Diversity of gasteroid fungi (Agaricomycetes, Basidiomycota) from the Brazilian Pampa Biome. Nova Hedwigia 106: 305-324. 